\title{
Performance assessment of demand controlled ventilation controls concerning indoor VOC exposure based on a dynamic VOC emission model
}

\author{
Klaas De Jonge ${ }^{l, *}$, Arnold Janssens ${ }^{l}$, and Jelle Laverge ${ }^{l}$ \\ ${ }^{1}$ Research group of Building Physics, Construction and Climate Control, Ghent University, Belgium \\ *Klaas.DeJonge@UGent.be
}

\begin{abstract}
The performance assessment of ventilation systems often focusses only on $\mathrm{CO}_{2}$ and humidity levels. The indoor Volatile Organic Compounds (VOC) emissions of building materials or other products is thereby overlooked. The new generation of ventilation systems, Demand Controlled Ventilation (DCV), are systems that do not supply the nominal airflow continuously but are controlled by $\mathrm{CO}_{2}$ or humidity sensors in order to save energy. This poses potential problems for exposure to VOCs. In this study, a dynamic VOC model, which takes into account changing temperature and humidity that was derived from literature, is implemented in a CONTAM model of the Belgian reference apartment. The impact of a DCV system on the indoor VOC levels is investigated. Results show that the use of a dynamic model is necessary compared to the previously used approximation of a constant emission. Furthermore, on a system level, the influence of the ventilation system control on the indoor VOC levels shows. The overall VOC concentration in the different rooms will be higher because of lowered ventilation rates. Especially in rooms that are often unoccupied during the day, the accumulation of VOCs shows. In the development of DCV system controls, the aspect of VOC exposure should not be overlooked to be able to benefit from both the energy savings and improved Indoor Air Quality (IAQ).
\end{abstract}

\section{Introduction}

In the performance assessment of Demand Controlled Systems (DCV), often, only the $\mathrm{CO}_{2}$ level and humidity are evaluated as they are indicators of comfort. However, it is well known that building materials, furniture and many other products emit Volatile Organic Compounds (VOCs) depending on temperature, humidity and age. These emissions can potentially result in long exposure to concentrations exceeding health guidelines.

DCV systems, which do not supply the nominal airflow continuously, carry the risk of letting VOC concentrations accumulate to higher levels during periods of lowered ventilation rate. With significant health risks correlated to often occurring VOCs, not taking into account VOC emissions in the assessment of a ventilation system or system control could lead to repeated situations of exposure to unhealthy indoor air [1], [2].

In simulations, VOCs are typically taken into account by assuming constant emissions based on the emission rate after 3, 7 or 28 days in a small-chamber emission test [3]. This gives a misrepresentation of real VOC emissions and VOC concentrations in the indoor environment. A temperature, humidity and time dependent model for the emissions of formaldehyde from Medium-Density Fiberboard (MDF) was used in this research to represent VOC emissions from indoor sources[4].

With this VOC emission model implemented in a CONTAM airflow model [5], four ventilation system strategies were simulated. A Belgian standard mechanical exhaust system (system $\mathrm{C}$, non-mechanical supply and mechanical exhaust), a balanced ventilation system (system D, both mechanical supply and exhaust) and a $\mathrm{DCV}$ variation of both systems referred to as system $\mathrm{C}+$ and system D+ respectively [6].

\section{Method}

Recent research led to models for a temperature dependent diffusivity, $\mathrm{D}_{\mathrm{m}}[7]$ and a humidity and 
temperature dependent starting concentration $\mathrm{C}_{\mathrm{o}}$ [8]. These correlations were introduced in the simplified emission equation from Xiong et al. [9] resulting in a temperature and humidity dependent emission model. The modelled contaminant is formaldehyde from $12 \mathrm{~mm}$ MDF. Assuming that every room has a similar source strength per square meter, the MDF is assumed to be used as flooring material in the rooms.

The used CONTAM model is an adaptation of the model developed by Heijmans et al. [10] and has been used in previous research concerning the Belgian NBN D50-001 ventilation standard [11]-[14]. The modelled home is the Belgian reference apartment and is shown in Figure 1.

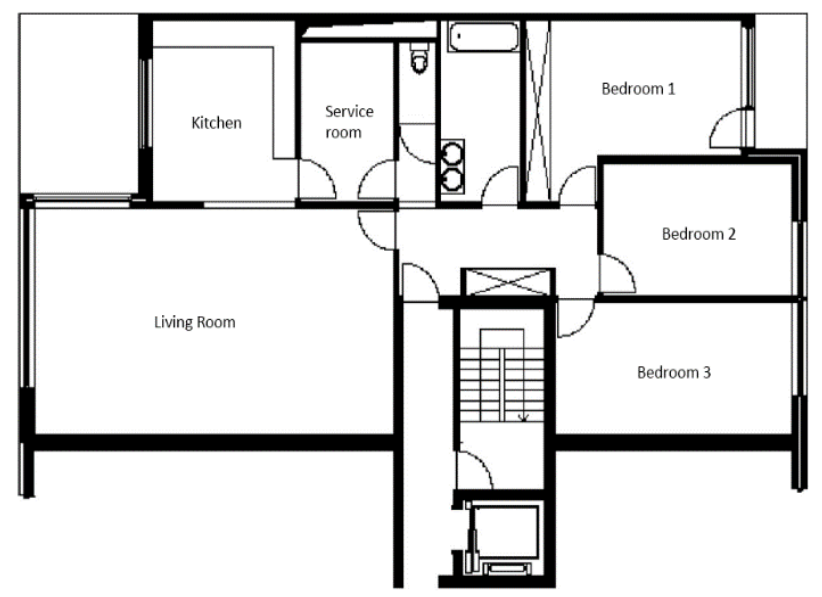

Figure 1. Floor plan of the Belgian reference apartment.

The DCV system that was chosen for this investigation is a system which combines $\mathrm{CO}_{2}$ sensors in spaces where air is supplied and humidity sensors in the spaces where air is extracted. If the component is mechanical, lowering the ventilation flow rates can be compared with altering the fan power. For the trickle ventilators of the mechanical exhaust DCV system $(\mathrm{C}+)$ ventilation, the inlet will open or close according to the needed flow rate.

For spaces where air is supplied (living room, bedrooms), there will be a linear correlation between the $\mathrm{CO}_{2}$ level in each room and the airflow rate with a minimum of $10 \%$ of the nominal airflow rate at the ambient $\mathrm{CO}_{2}$ concentration. As the scope of this research are the indoor pollutants, ambient $\mathrm{CO}_{2}$ levels are assumed constant at $350 \mathrm{ppm}$. This concentration is also assumed in the current assessment method for demand controlled ventilation and is therefore adopted in this study. As ambient concentrations nowadays are often higher than $400 \mathrm{ppm}$, the assumed ambient concentrations should be revised in the future.

For spaces where air is extracted (kitchen, toilet, bathroom), the system is based on a centralized mechanism. It will adapt the extraction flow rates of all exhaust components according to the space with the highest Relative Humidity (RH). The extraction airflow rates are linearly correlated to the relative humidity with an the upper and lower limit set at $\mathrm{RH}=35 \%$ and $\mathrm{RH}=70 \%$ respectively. Table 1 shows the control values of the DCV systems.

Table 1. Control values for DCV system.

\section{Supply flow rate}

\begin{tabular}{|c|c|c|}
\hline \multirow{2}{*}{ Living room } & \multicolumn{2}{|c|}{$\mathrm{CO}_{2}$} \\
\cline { 2 - 3 } Bedroom 1 & $3.0 \mathrm{l} / \mathrm{s}$ & $30 \mathrm{l} / \mathrm{s}$ \\
Bedroom 2 & $1.21 / \mathrm{s}$ & $121 / \mathrm{s}$ \\
Bedroom 3 & $1.01 / \mathrm{s}$ & $10 \mathrm{l} / \mathrm{s}$ \\
& $1.11 / \mathrm{s}$ & $111 / \mathrm{s}$
\end{tabular}

Exhaust flow rate

\begin{tabular}{l|c|c|}
\hline \multirow{2}{*}{} & \multicolumn{2}{|c|}{ Relative Humidity* } \\
\multirow{2}{*}{$\begin{array}{l}\text { Total exhaust } \\
\text { flow rate }\end{array}$} & $5.81 / \mathrm{s}$ & $58 \mathrm{1} / \mathrm{s}$ \\
\cline { 2 - 3 } & &
\end{tabular}

* Lowest relative humidity measured in: kitchen, bathroom, service room or toilet

\section{Results}

The results of the simulations support the need for a dynamic VOC emission model.

For all cases, using a constant emission model would lead to biased results about the real VOC concentrations and will lead to an inaccurate assessment of the examined ventilation system. Figure 2 shows that using a constant emission model in the performance assessment will overestimate the exposures during the summer months and underestimate them during the winter months, regardless of the used ventilation system. Additionally, peak values for the more humid spaces of the house are severely underestimated in both value and occurrence.

Further investigation clearly showed the difference between continuous ventilation systems and DCV systems. When comparing the two graphs of Figure 3, it is clear that the VOC concentration in the rooms has a large dependency on the chosen ventilation system control. For the standard balanced ventilation system (D), The lowest levels will be in the rooms with air supply and highest in rooms with exhaust components. For the balanced DCV system $(\mathrm{D}+$, ) this is not necessarily the case. 

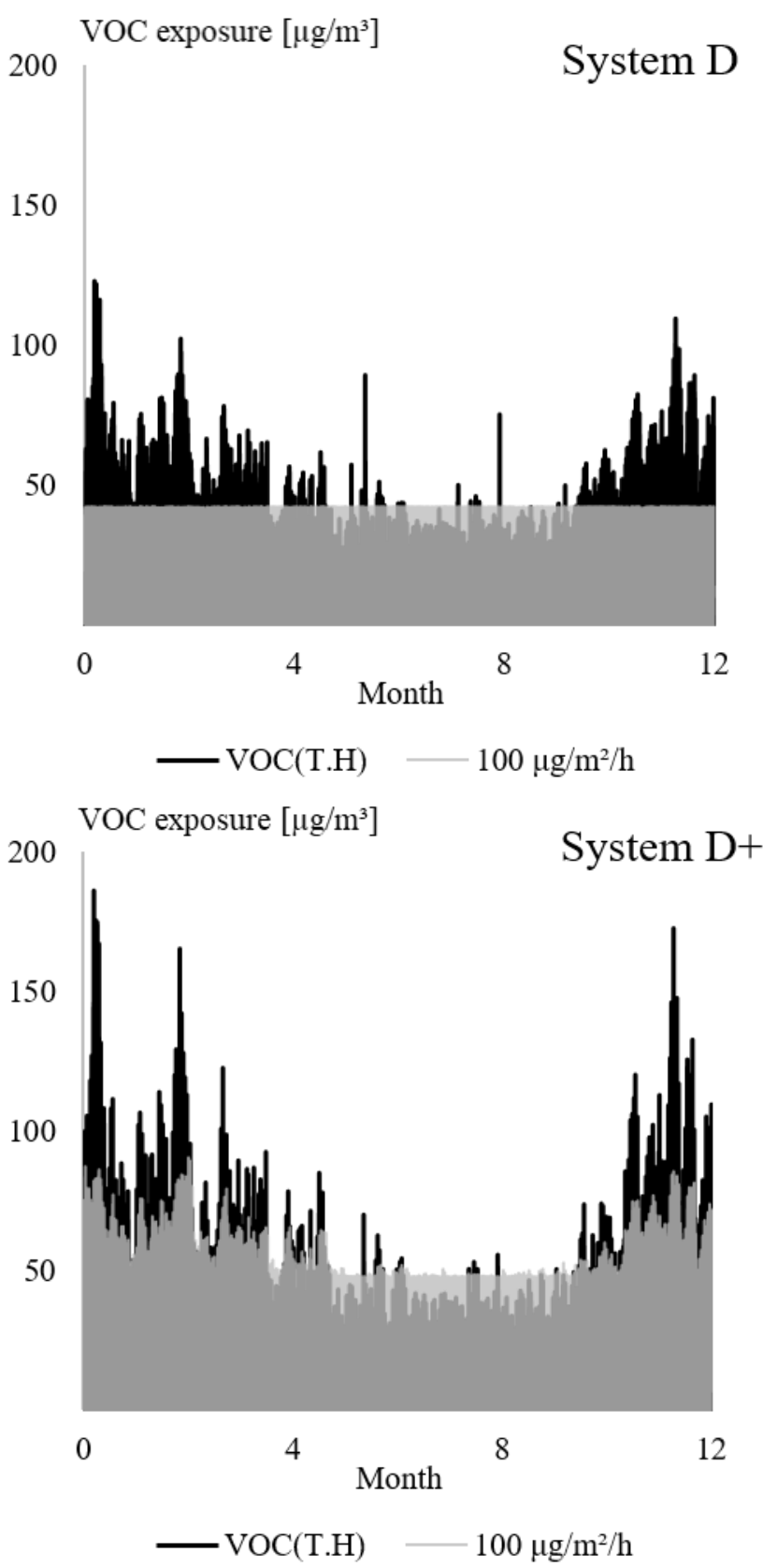

Figure 2. Balanced DCV system, D+ (top) and standard balanced ventilation system D (bottom). Continuous VOC supply (grey) ' $100 \mu \mathrm{g} / \mathrm{m}^{2} / \mathrm{h}$ ' and temperature and humidity dependent VOC model (black) 'VOC(T.H)'.
Because people aren't present in some of the rooms during the day and thus no $\mathrm{CO}_{2}$ or humidity is produced in these rooms, the DCV system lowers the provided airflow rates accordingly. The expected higher accumulation of VOCs due to the more continuous character of the emissions clearly shows for spaces that are left unoccupied for longer periods of time.

As can be seen in Figures 3, for a DCV system, VOC levels rise in the bedrooms during the day whereas in the living room, VOC levels stay low.

For all cases, using a constant emission model would lead to biased results about the real VOC concentrations and will lead to an inaccurate assessment of the examined ventilation system. Figure 2 shows that using a constant emission model in the performance assessment will overestimate the exposures during the summer months and underestimate them during the winter months, regardless of the used ventilation system. Additionally, peak values for the more humid spaces of the house are severely underestimated in both value and occurrence.

Further investigation clearly showed the difference between continuous ventilation systems and DCV systems. When comparing the two graphs of Figure 3, it is clear that the VOC concentration in the rooms has a large dependency on the chosen ventilation system control. For the standard balanced ventilation system (D), The lowest levels will be in the rooms with air supply and highest in rooms with exhaust components. For the balanced DCV system $(\mathrm{D}+$, ) this is not necessarily the case.

Because people aren't present in some of the rooms during the day and thus no $\mathrm{CO}_{2}$ or humidity is produced in these rooms, the DCV system lowers the provided airflow rates accordingly. The expected higher accumulation of VOCs due to the more continuous character of the emissions clearly shows for spaces that are left unoccupied for longer periods of time.

As can be seen in Figures 3, for a DCV system, VOC levels rise in the bedrooms during the day whereas in the living room, VOC levels stay low. 

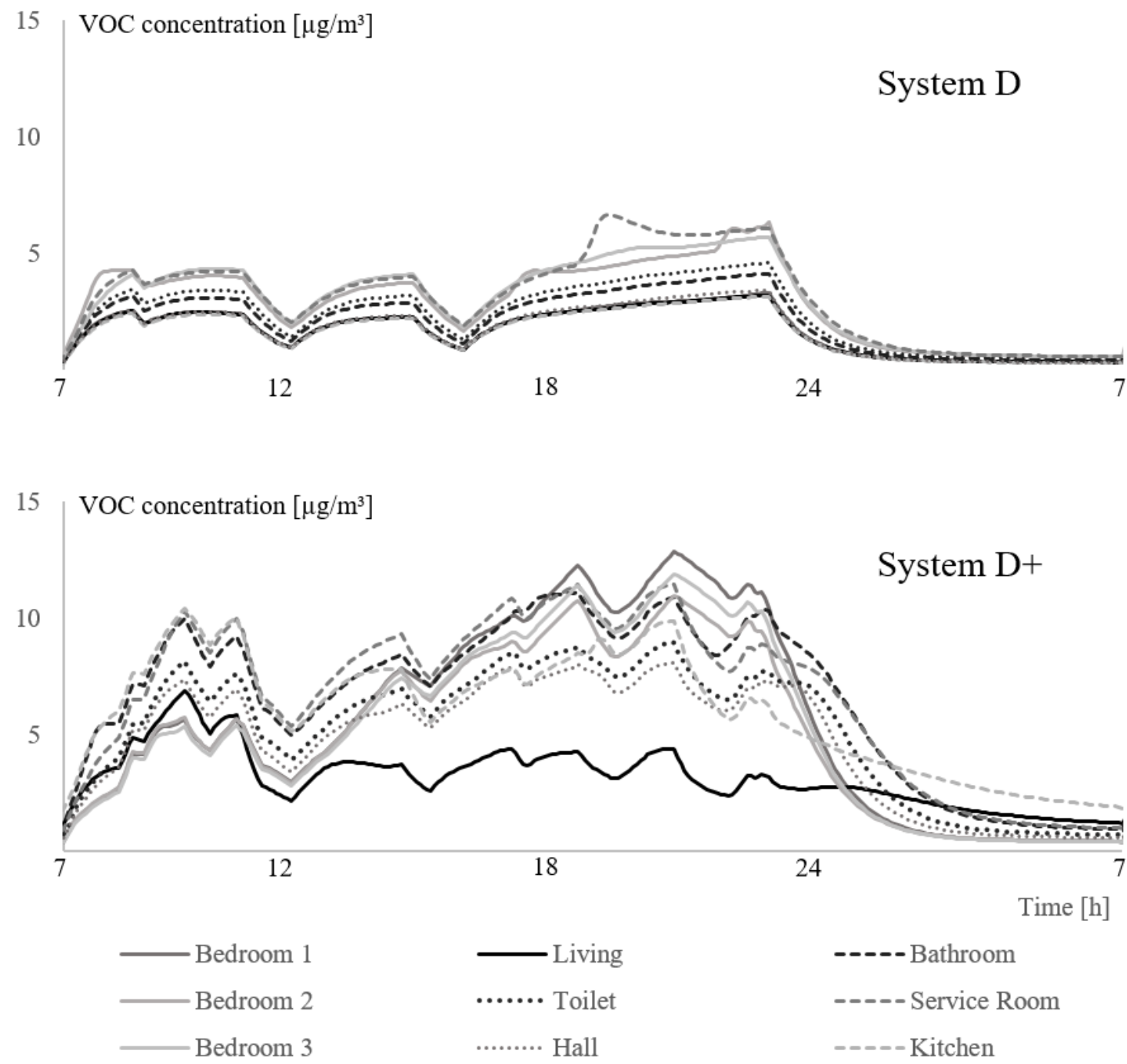

Figures 3. Indoor VOC concentrations during $24 \mathrm{~h}$ for the standard balanced ventilation system, D (top) and balanced DCV system, $\mathrm{D}+$ (bottom) showing overall higher VOC levels and accumulation of VOCs in unoccupied rooms.

\section{Discussion}

In these simulations, the minimum ventilation rate is only $10 \%$ of the nominal flow rate for all spaces. If this minimum ventilation rate would be higher in exhaust spaces and bedrooms, the VOC accumulation resulting in higher values would not be as significant. For the spaces where air is extracted, lowering the upper limit for the humidity control should have a similar positive influence.

Looking at the ventilation control factors influencing the VOC exposure, in comparison with the DCV system used in this research, it can be seen that only controlling the $\mathrm{CO}_{2}$ inlet flow rates but using continuous extraction will most likely lower the peak values to the same level as the normal system. This means the chances of exposure to concentrations exceeding health limits is lowered as well.
A higher potential risk lies in humidity controlled mechanical exhaust DCV systems (C). For these systems, exhaust airflow rates will be similar but inlet airflow rates will depend on the sometimes lowered pressure difference between the natural inlets and the mechanical exhausts. This, in combination with the unpredictable nature of a natural inlet, will result in overall higher VOC exposure concentrations.

Important to note is that if the average airflow rate is higher, the energy saving potential of the DCV system will be lower. Having both an energy efficient system and a good IAQ requires further investigation on the level of control strategies and their impact on IAQ. 


\section{Conclusion}

DCV systems will have a significant impact on the occupants exposure to VOCs. In DCV system design, the influence to indoor VOCs should be considered and assessed. To do so, a continuous emission model will not be adequate. Simulations done as part of this research clearly show an important dependency of indoor VOC levels to changing temperatures and humidity. Although only one control strategy is simulated for this study, it is clear that the way the DCV system is controlled can have more or less influence on indoor VOC levels.

For the simulated DCV system specifically, the total yearly dose of exposure of the simulated VOC will be approximately $10 \%$ higher than the continuous, standard ventilation system. If the goal is to have the same average exposure to VOCs on a yearly basis or have the same IAQ but use less energy, implementing additional control measures for VOCs will be necessary.

\section{References}

[1] T. Salthammer, S. Mentese, and R. Marutzky, "Formaldehyde in the Indoor Environment," Chem. Rev., vol. 110, no. 4, pp. 2536-2572, Apr. 2010.

[2] J. M. Logue, T. E. McKone, M. H. Sherman, and B. C. Singer, "Hazard assessment of chemical air contaminants measured in residences," Indoor Air, vol. 21, no. 2, pp. 92-109, Apr. 2011.

[3] K. D. Brouwere, S. Caillou, R. Torfs, and P. Van den Bossche, "Q-INTAIR: modellering van binnenluchtkwaliteit op basis van bouwmaterialenemissies, gebouwluchtdichtheid en ventilatie van woningen," Sep. 2009.

[4] K. De Jonge, "The impact of demand controlled ventilation on indoor VOC exposure in Belgian dwellings," Master dissertation, Ghent University, 2018.

[5] W. S. Dols and B. J. Polidoro, "CONTAM User Guide and Program Documentation Version 3.2," National Institute of Standards and Technology, NIST TN 1887, Sep. 2015.

[6] NBN D50-001, "Ventilatievoorzieningen in woongebouwen," Jan. 1991.

[7] Q. Deng, X. Yang, and J. Zhang, "Study on a new correlation between diffusion coefficient and temperature in porous building materials," Atmos. Environ., vol. 43, no. 12, pp. 2080-2083, Apr. 2009.

[8] W. Liang, M. Lv, and X. Yang, "The combined effects of temperature and humidity on initial emittable formaldehyde concentration of a medium-density fiberboard," Build. Environ., vol. 98, pp. 80-88, Mar. 2016.

[9] J. Xiong, W. Wei, S. Huang, and Y. Zhang, "Association between the Emission Rate and Temperature for Chemical Pollutants in Building Materials: General Correlation and
Understanding," Environ. Sci. Technol., p. 130709124156006, Jul. 2013.

[10] N. Heijmans, N. Van Den Bossche, and A. Janssens, "Berekeningsmethode gelijkwaardigheid voor innovatieve ventilatiesystemen in het kader van de EPB-regelgeving," WTCB, Ghent University, Mar. 2007.

[11] J. Laverge and A. Janssens, "Optimization of design flow rates and component sizing for residential ventilation," Build. Environ., vol. 65, pp. 81-89, Jul. 2013.

[12] J. Laverge and A. Janssens, "Residential ventilation system optimization using Monte-Carlo and genetic algorithm techniques," in ASHRAE IAQ, 16th Conference, Proceedings, 2010.

[13] A. Janssens, L. Willems, and J. Laverge, "Performance evaluation of residential ventilation systems based on multi-zone ventilation models.," p. 7, 2008 .

[14] S. Caillou, N. Heijmans, J. Laverge, and A. Janssens, "Méthode de calcul PER: Facteurs de réduction pour la ventilation à la demande," p. 141, 2014. 